\title{
Medical informatics applications in COVID-19 crisis control: Protocol for systematic literature review
}

\author{
Saeid Eslami1,2,3(i), Raheleh Ganjali 1,4*(i) \\ ${ }^{1}$ Department of Medical Informatics, Faculty of Medicine, Mashhad University of Medical Sciences, Mashhad, Iran \\ ${ }^{2}$ Pharmaceutical Research Center, Mashhad University of Medical Sciences, Mashhad, Iran \\ ${ }^{3}$ Department of Medical Informatics, University of Amsterdam, Amsterdam, Netherlands \\ ${ }^{4}$ Clinical Research Development Unite, Emam Reza Hospital, Mashhad University of Medical Sciences, Mashhad, Iran
}

\begin{tabular}{|c|c|}
\hline Article Info & A B S T R A C T \\
\hline $\begin{array}{l}\text { Article type: } \\
\text { Review }\end{array}$ & $\begin{array}{l}\text { Introduction: SARS-CoV-2 has disseminated globally, and COVID-19 has } \\
\text { been labeled as a public health emergency of global concern by the World } \\
\text { Health Organization. Since } 2019-\mathrm{nCoV} \text { ( } 2019 \text { new coronavirus) has a long }\end{array}$ \\
\hline $\begin{array}{l}\text { Article History: } \\
\text { Received: } 2020-12-29 \\
\text { Accepted: } 2021-01-15 \\
\text { Published: } 2021-01-18\end{array}$ & $\begin{array}{l}\text { incubation period and high infectivity, e-Health and its subsets in medical } \\
\text { informatics have evolved as a suitable solution to enable the continuity of } \\
\text { health services delivery. Also, new health care models are required during } \\
\text { the COVID-19 pandemic. The proposed systematic review aims to examine } \\
\text { and summarize evidence related to medical informatics applications in }\end{array}$ \\
\hline $\begin{array}{l}\text { *Corresponding author: } \\
\text { Raheleh Ganjali }\end{array}$ & \\
\hline $\begin{array}{l}\text { Clinical Research Development } \\
\text { Unite, Mashhad University of } \\
\text { Medical Sciences, Mashhad, Iran }\end{array}$ & $\begin{array}{l}\text { Material and Methods: A research team consisting of experts in the fields } \\
\text { of medical informatics and systematic review methods were guided this } \\
\text { review according to the Cochrane Handbook and PRISMA reporting } \\
\text { guidelines. PubMed and Scopus databases were searched. Eligibility criteria }\end{array}$ \\
\hline Email:Ganjalir2@mums.ac.ir & $\begin{array}{l}\text { for including studies reviewed was randomized and non-randomized } \\
\text { controlled trials published in English language. Articles performed on } \\
\text { medical informatics applications in COVID-19 pandemic during 2019-2020 }\end{array}$ \\
\hline $\begin{array}{l}\text { Keywords: } \\
\text { Medical Informatics } \\
\text { COVID-19 } \\
\text { Systematic Review } \\
\text { Quality Assessment }\end{array}$ & $\begin{array}{l}\text { were identified. Two independent reviewers will assess articles eligibility } \\
\text { and extract data into a spreadsheet using a structured pilot-tested form. } \\
\text { Collected data and evidence will be synthesized using a thematic synthesis } \\
\text { approach. The risk of bias will be assessed in all included studies using } \\
\text { appropriate tools. }\end{array}$ \\
\hline
\end{tabular}

Results: The literature search led to the identification of a total of 1882 and 854 articles retrieved from the PubMed and Scopus databases, respectively. After removing duplicates, 2716 articles remained and underwent title and abstract screening process. The results of this review are expected to be served as a basis for assisting researchers, decision makers, medical informatics specialists, politicians, and others in developing, implementing, and evaluating IT-based tools and interventions to help medical staff in combating and eradicating COVID-19.

Conclusion: This systematic review is the first comprehensive evaluation of MI methods aiming to control and manage covid-19 pandemic. This study highlights applications of medical informatics in pandemic situation and will help future researchers to take the most advantage of using MI in the health system.

Cite this paper as:

Eslami S, Ganjali R. Medical Informatics Applications in COVID-19 Crisis Control: Protocol for Systematic Literature Review. Front Health Inform. 2021; 10: 56. DOI: 10.30699/fhi.v10i1.272

\section{INTRODUCTION}

In 2019, a novel coronavirus called Severe Acute Respiratory Syndrome Coronavirus 2 (SARS-CoV-2) caused the international outbreak of COVID-19 (Coronavirus Disease 2019) respiratory illness [1].
SARS-CoV-2 has disseminated globally, and COVID19 has been labeled as a public health emergency of global concern by the World Health Organization [2]. Principal modes of spread reported include diffusion through respiratory droplets and possible diffusion 
through airborne particles. It is worth noting that the virus could also be transmitted during its incubation period by asymptomatic carriers [픈 $\underline{4}$. Since 2019nCoV (2019 new coronavirus) has a long incubation period and high infectivity [ㄷ], the prevention and control of the COVID-19 pandemic face major challenges. To ensure safety in health care delivery and meet patients' needs, health systems have adjusted care processes and employed new approaches to screen and care for COVID patients so that routine health care delivery could continue and meet patients' needs []ㅡ.

In this scenario, e-Health has evolved as a suitable solution to enable the continuity in health services delivery []. E-Health is generally described as the provision of health-associated services through the safe and cost-effective application of information and communication technologies (ICTs) []ㅡ. E-Health solutions could support care and treatment through exchanging treatment data among health care providers or between patients and health care providers not complying with data protection regulations and also through providing documents appropriate for tele-intensive care needs of remote COVID-19 patients [9]. Due to the high transmission rate of COVID-19, most countries have imposed strict lockdowns and curfews; hence, patient-physician communication and visits are challenging. In the era of digital health technologies, the focus on developing new models has shifted towards telehealth (virtual visits, virtual care), mobile apps (remote patient monitoring), websites, and chatbots (risk assessment, screening, triage) [10]. It is clear that health information technology has played significant roles in the combat against COVID-19 so far. However, new health care models are required during the COVID-19 pandemic []].

Incorporation of new health technologies and practices such as big data, artificial intelligence (AI), telehealth services, health information exchange (HIE) services and mobile health applications [ㅇ] into health care systems could assist health care providers to deal with this global challenge in different ways. Incorporation of big data, such as transportation data and location-based services data, could be useful in modelling viral activity and providing guidance for health care policymakers; artificial intelligence (AI) and deep learning could also be beneficial in improving the detection and diagnosis of COVID-19 and facilitating the development of novel drugs [11]. All of these methods are useful for providing strong support in the pandemic prevention and control [12].

Many studies have described and examined different dimensions of Medical Informatics (MI) in the control and management of COVID-19 [10-13]. Thus, the aim of this systematic review study is to explain and summarize the applications of medical informatics in
COVID-19 crisis by evidence. The results of which may be served as an evidence-based yardstick in the development and implementation of health information technology innovations.

The use of health technologies in research and judgment about the pandemic causes innovations in the diagnosis and therapy, improves services efficiency, and strengthens the capacity of information technology to support the pandemic prevention and control. We will raise research questions and define the study population, the type of intervention applied, the control used to compare the results, and the outcome framework used to identify and combine Medical Subject Headings, subject headings, and keywords.

\section{Research questions}

The aim of this review is to identify applications of medical informatics in COVID-19 crisis. The impact of IM would be described in terms of one or more of the three metrics. The research questions addressed are as follows:

- Which studies have used different medical informatics tools in COVID-19 crisis?

- What are the applications of medical informatics in these studies?

- What are the most commonly used methods in these studies?

- How is the quality of these studies?

The participants in all studies evaluated are human subjects. The intervention is medical informatics tools, and the control is conventional health care methods. A positive outcome is identified if medical informatics assists the health system in diagnosing, treating (including follow up), and/or controlling pandemic.

\section{MATERIAL AND METHODS}

The Cochrane protocol guide was used to develop this systematic review protocol [14]. The Preferred Reporting Items for Systematic Reviews and MetaAnalysis (PRISMA) Protocols (PRISMA-P) 2015 Checklist [15] was employed to report this systematic review protocol. The research team consists of healthcare and MI professionals who are skilled in research and teaching and have clinical experience in medical informatics. SE and RG have conducted extensive research in the field of systematic review as well as design and evaluation of information technology-based interventions. SE has a multidisciplinary background in pharmacology and medical informatics. Also, RG has multidisciplinary background in health information technology and medical informatics. SE has also conducted extensive research and has teaching experience in medical 
informatics.

\section{Eligibility criteria}

\section{Study design}

Studies considered as eligible for inclusion in this review include quantitative and qualitative studies and studies conducted with randomized and nonrandomized study designs. Non-randomized studies include case-control, cohort, and cross-sectional studies in which MI is the primary intervention used. Randomized studies include randomized controlled trials and quasi experimental studies.

\section{Study participants}

All studies describing the use of MI targeted at assisting health care providers to diagnose, treat, manage, and control COVID-19 disease among individuals or groups will be considered as eligible for inclusion in this research. Study participants will be people with different ages, genders, ethnicities, occupations, and roles, included in the reviewed studied (i.e. patients and health professionals).

\section{Intervention and control}

Medical informatics is the interconnection of information technology and different disciplines of medicine to improve health care and treatment outcomes. Medical informatics emphasizes the use of technology as an essential tool targeted to assist in organizing, analyzing, managing, and using information [16]. According to American medical informatics association (AMIA), "Medical informatics has to do with all aspects of understanding and promoting the effective organization, analysis, management, and use of information in health care" [16].

Interventions intended to be evaluated in the study should aim to strengthen the health system by MI in combating COVID-19 disease. MI literature could be divided into three subdivision: 1) the organization, application, and evaluation of health information systems, 2) medical knowledge representation, and 3) signal and data analysis [17].

Controls may consist of other routine care or advanced technologies traditionally adopted in health systems to enhance diagnosis, treatment, and control of the disease as well as decision making about the pandemic.

\section{Outcomes}

In fact, studies investigating at least one outcome will be included in this review. The outcomes will be categorized into two groups: clinical and process outcomes. Clinical outcomes are biomarkers demonstrating the disease severity, such as blood pressure. Care process outcomes are defined as the outcomes affecting patients' care by improving the quality of care and interaction between care providers and patients [18]. Studies focusing on the development, applicability, or description of IT-based instruments without evaluating any outcomes will be excluded.

\section{Search Methods}

Electronic databases, including PubMed and Scopus, were systematically searched. Following exploratory research around the research questions, Medical Subject Headings, subject headings, and keywords were identified. No restriction was applied regarding the publication date of studies included. However, only studies published in English was included. PubMed is intended to be first searched by implementing a search strategy for a preliminary research. Based on the findings of this search, the search strategy were developed and adapted for searching the Scopus database. The search were restricted by date from 2019 to the end of September 19, 2020.

\section{Search strategy}

The search terms were be identified based on two key concepts, including medical informatics and related concepts (behavior or phenomenon of interest) as well as COVID-19 (health context). Keywords and Mesh terms related to each of these concepts were searched in the literature and thesauruses (Table 1). Both Medical Subject Headings and free-text terms were searched. Truncation and adjacency searching was used to increase the sensitivity of the search as much as possible.

\section{Data management}

EndNote X8.2 (Clarivate Analytics, Philadelphia, PA, USA) was used to manage the results of our searches and remove duplicates. Duplicates not identified by the Endnote were manually removed. The bibliographic citations of studies included were also be manually searched to identify other studies that meet the inclusion criteria. Studies selection was done with human.

\section{Study selection}

Titles and abstracts of selected studies will be screened for eligibility by two independent reviewers based on the inclusion criteria. Contradictions will be resolved through discussion between two reviewers and, if needed, by the Judgment and intervention of a third independent reviewer. The full text of all studies selected during the screening process will be reviewed independently by two reviewers, and any disagreement will be resolved as described earlier. A PRISMA flow chart will be prepared to show the details and steps of the selection process [19]. 
Table 1: Keywords and Mesh terms used in the search strategy

\begin{tabular}{|c|c|c|}
\hline & Mesh Terms & Other Terms \\
\hline 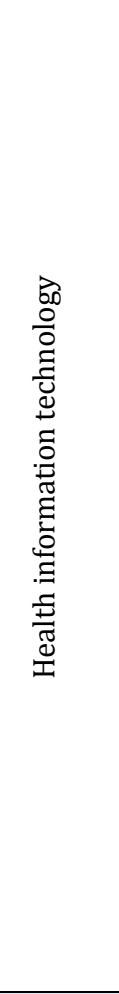 & $\begin{array}{l}\text { Telecommunications, telemedicine, } \\
\text { computers, handheld, medical } \\
\text { informatics } \\
\text { hospital medication systems, } \\
\text { adverse drug reaction reporting } \\
\text { systems, radiology information } \\
\text { systems, clinical laboratory } \\
\text { information systems, electronic health } \\
\text { records, electronic prescribing, } \\
\text { computerized medical records } \\
\text { systems, hospital information systems, } \\
\text { medical informatics applications, } \\
\text { expert systems } \\
\text { medical order entry systems, clinical } \\
\text { decision support systems, decision } \\
\text { support system management, decision } \\
\text { support techniques, point-of-care } \\
\text { systems, information storage and } \\
\text { retrieval software, patient portals, } \\
\text { health information exchange and } \\
\text { monitoring system, smartphone } \\
\text { cellular phone mobile, cell phone } \\
\text { mobile applications, web browser, } \\
\text { internet web information technology, } \\
\text { information systems }\end{array}$ & $\begin{array}{l}\text { Telemetry, mobile health, m-health, telehealth, e-health, personal } \\
\text { digital, assistant, PDA computer, handheld computer, palm-top } \\
\text { computer, computer, tablet, health informatics, clinical informatics, } \\
\text { health information technology, medical information science, hospital } \\
\text { unit dose drug distribution systems, medication hospital systems, } \\
\text { adverse drug reaction reporting systems, picture archiving and } \\
\text { communication systems, system, X-ray information, clinical } \\
\text { laboratory information systems, laboratory information system, } \\
\text { electronic medical record, computerized medical record, electronic } \\
\text { health record, E-prescribing, electronic prescription, E-prescription, } \\
\text { automated medical records system, computerized medical records } \\
\text { system, automated medical record system, multi-hospital } \\
\text { information systems, informatics applications, medical, medical } \\
\text { informatics application, expert systems, medication alert system, } \\
\text { medication system*, medication alert/reminder system, } \\
\text { computerized physician order entry system, computerized provider } \\
\text { order entry system, CPOE, decision analyses, decision modeling, } \\
\text { clinical prediction rule, prediction rule, clinical prediction, decision } \\
\text { analysis, decision analyses, point of care technology, information } \\
\text { extraction, computer program, software tool, computer software } \\
\text { application, computer programs and programming, patient web } \\
\text { portal, patient internet portals, patient portal, medical information } \\
\text { exchange*, health information exchange*, screening system, } \\
\text { surveillance system, smart phone*, cellular phone, mobile phone, } \\
\text { transportable cellular phone, mobile app, portable electronic app, } \\
\text { world wide web, ancillary information system, emergency care } \\
\text { information system }\end{array}$ \\
\hline $\begin{array}{l}\text { Corona } \\
\text { virus }\end{array}$ & $\begin{array}{l}\text { Severe acute respiratory syndrome } \\
\text { coronavirus } 2\end{array}$ & $\begin{array}{l}\text { Wuhan coronavirus, Wuhan seafood market pneumonia virus, } \\
\text { COVID-19 disease, coronavirus disease 2019, SARS-CoV-2, SARS 2, } \\
\text { 2019-nCoV, } 2019 \text { novel coronavirus }\end{array}$ \\
\hline
\end{tabular}

\section{Data extraction}

Two reviewers will independently extract data in each eligible study using a pre-piloted Microsoft Excel data extraction form. The third reviewer will review the extracted data to ensure the accuracy and completeness of the data. The following information will be extracted from the included studies: author(s), year and month of publication, country, study aim, study design, sample size, method used, outcomes, result, and conclusion.

\section{Quality assessment}

The quality of the included studies will be assessed by two authors using appropriate instruments selected based on the type of each article. In case of disagreement, the consensus will be sought by the third reviewer. The quality of observational articles (cohort, cross-sectional, and case-control) will be evaluated using the STROBE tool [20]. This tool quantifies the risk of bias in terms of 22 sections as follows: title, abstract, background/rationale, objectives, study design, setting (place and time), participants (in two parts of method and result), variables, data sources/ measurement, bias, study size, quantitative variables, statistical methods, descriptive data, outcome data, main results, other analyses, key results, limitations, interpretation, generalizability, and funding. Each item is scored either by $0,0.5$, or 1 , indicating that article doesn't contain relevant content, contains relevant content with no elaboration, and contains relevant content with elaboration, respectively.

The quality of prediction studies will be assessed using the Prediction model Risk of Bias Assessment Tool (PROBAST) [21] which is applied to determine the applicability and risk of bias in diagnostic and prognostic studies. PROBAST includes 20 signaling questions in four areas as follows: participant selection, predictors, outcome, and analysis. Each area is scored as low, high, or unclear. The quality of quasi-experimental studies will be assessed by applying the Quality Assessment Tool designed by Brown based on the study of Estabrooks et al. (2001, 2009) for pre and post intervention study designs $[\underline{22}, \underline{23}]$. This tool assesses six areas including sampling, design, control of confounders, data collection and outcome measurement, statistical analysis, and conclusions and dropouts.

Finally, the Cochrane Collaboration instrument will be applied to evaluate the risk of bias in clinical trials [14]. This tool has been designed to evaluate the risk 
of bias in terms of the following domains: selection bias (i.e. random sequence generation and allocation concealment), reporting bias (selective outcomes reporting), performance bias (i.e. blinding of participants and personnel), attrition bias (incomplete outcomes data), detection bias (blinding of outcome assessor), etc.

Each study will be evaluated independently by two reviewers, and in case of any disagreement, a bilateral discussion session will be hold to reach a consensus.

\section{Data synthesis}

The applications of MI will be identified using a thematic synthesis approach comprising three stages as follows [24]. At first, the themes of the selected articles are identified through a series of meetings after summarizing and classifying the results, which appeared to be relevant, into higher-order categories. Classifying is undertaken to develop more understandable and essential topics from recurrent themes or a varied number of results. In the second stage, the codes are grouped into categories based on their similarities and differences, and a descriptive theme is created to capture the meaning of each category. In the final stage, analytical themes are generated from descriptive themes. Analytical themes are interpretations of descriptive themes, which usually go beyond the findings of the original studies. Analytical themes are inferred from descriptive themes based on the observed interrelationship with the definitions of MI dimensions. All the reviewers will initially generate analytical themes independently and then collectively in group in order to minimize the risk of bias [24].

\section{RESULTS}

In this review study, all included studies were published from 2019 to 2020 concurrent with COVID-19 outbreak. The literature search led to the identification of a total of 1882 and 1045 articles retrieved from the PubMed and Scopus databases, respectively (Fig 1). Then databases search results were imported to Endnote. 211duplicate articles were removed. After removing duplicates, 2716 articles remained and underwent title and abstract screening process.

The results of this systematic review provide a comprehensive view of various applications of MI in COVID-19. The present study results are expected to be served as a basis for assisting researchers, decision makers, MI specialist, politicians, and others in developing, implementing, and evaluating ITbased tools and interventions to help medical staff in combating and eradicating COVID-19.

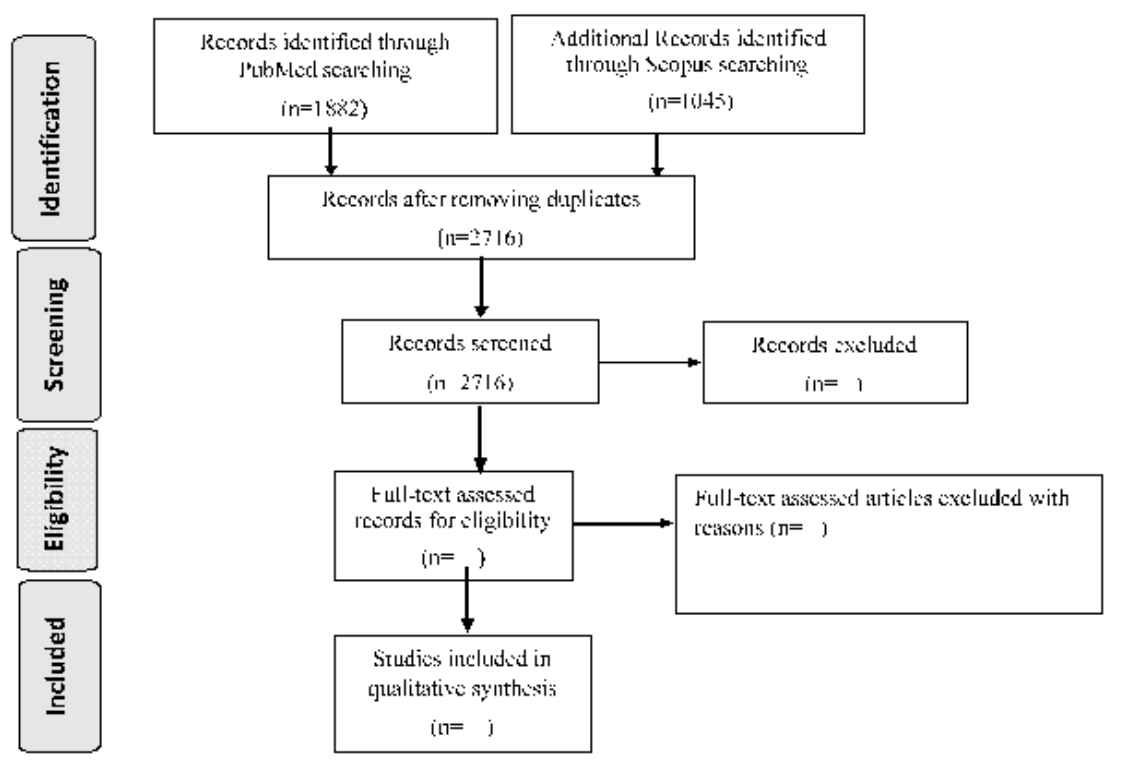

Fig 1: Flow diagram of the literature search and publications selection

\section{DISCUSSION}

This systematic review aims to identify the applications of medical informatics in COVID-19 pandemic. To the best of our knowledge, this review is the first attempt undertaken to develop an evidence-based method using a systematic review approach. The proposed systematic review will introduce further research and innovations in terms of advantages and disadvantages of using MI in the health system. The primary aim of this review is to determine whether MI could assist health care providers in providing health services to improve the 
diagnosis, treatment, management, and control of COVID-19 as well as to determine the most common MI-based methods used in COVID-19 pandemic.

Other studies were done in organ transplant and lung transplant $[\underline{25}, \underline{26}]$. The use of a wide range of keywords and Mesh terms to develop the search strategy may also be considered as a strength point of this review. However, the generation of analytical themes from descriptive themes through thematic synthesis is considered as controversial because it is affected by the insight and judgment of the reviewers, but multidisciplinary perspectives and the extensive experience of the reviewers are believed to increases the value of synthesized data in this study. Finally, it is expected that the use of a transparent and rigorous systematic review approach in this study will result in the development of an evidence-based approach to the use MI.

\section{CONCLUSION}

To the best of our knowledge, this systematic review

\section{REFERENCES}

1. Juul S, Nielsen EE, Feinberg J, Siddiqui F, Jørgensen CK, Barot E, et al. Interventions for treatment of COVID19: A living systematic review with meta-analyses and trial sequential analyses (The LIVING Project). PLoS Med. 2020; 17(9): e1003293. PMID: 32941437 doi: 10.1371/journal.pmed.1003293 [PubMed]

2. World Health Organization. Novel Coronavirus (2019nCoV): Situation report-3 [Internet]. 2020 [cited: 1 Oct 2020]. Available from: https://www.who.int/docs/defaultsource/coronaviruse/situation-reports/20200123sitrep-3-2019-ncov.pdf?sfvrsn=d6d23643_8

3. Gavriilidis P, Pai M. The impact of COVID-19 global pandemic on morbidity and mortality of liver transplant recipients children and adults: A systematic review of case series. J Clin Med Res. 2020; 12(7): 404-8. PMID: 32655733 DOI: 10.14740/jocmr4223 [PubMed]

4. Guan W-j, Ni Z-y, Hu Y, Liang W-h, Ou C-q, He J-x, et al. Clinical characteristics of coronavirus disease 2019 in China. N Engl J Med. 2020; 382: 1708-20.

5. Peeri NC, Shrestha N, Rahman MS, Zaki R, Tan Z, Bibi $S$, et al. The SARS, MERS and novel coronavirus (COVID-19) epidemics, the newest and biggest global health threats: What lessons have we learned? Int J Epidemiol. 2020; 49(3): 717-26. PMID: 32086938 DOI: 10.1093/ije/dyaa033 [PubMed]

6. Mehrotra A, Chernew M, Linetsky D, Hatch H, Cutler D. The impact of the COVID-19 pandemic on outpatient visits: A rebound emerges [Internet]. 2020 [cited: 1 Oct 2020]. Available from: https://www.commonwealthfund.org/publications/ 2020/apr/impact-covid-19-outpatient-visits is the first comprehensive evaluation of MI methods aiming to control and manage covid-19 pandemic. This study highlights applications of medical informatics in pandemic situation and will help future researchers to take the most advantage of using MI in the health system.

\section{AUTHOR'S CONTRIBUTION}

The authors agree on this final form of the manuscript, and attested that all authors contributed in the final draft of the manuscript.

\section{CONFLICTS OF INTEREST}

The authors declare no conflicts of interest regarding the publication of this study.

\section{FINANCIAL DISCLOSURE}

This study was supported by a grant from Mashhad University of Medical Sciences (990447) Research Council.

7. Dores AR, Geraldo A, Carvalho IP, Barbosa F. The use of new digital information and communication technologies in psychological counseling during the CoViD-19 pandemic. Int J Environ Res Public Health. 2020; 17(20): 7663. PMID: 33096650 DOI: 10.3390/ijerph17207663 [PubMed]

8. World Health Organization. Fifty-eighth world health assembly: Resolutions and decisions annex. Geneva: WHO; 2005.

9. Tebeje TH, Klein J. Applications of e-Health to support person-centered health care at the time of COVID-19 pandemic. Telemed J E Health. 2020. PMID: 32746750 DOI: $10.1089 / \mathrm{tmj} .2020 .0201$ [PubMed]

10. Fagherazzi G, Goetzinger C, Rashid MA, Aguayo GA, Huiart L. Digital health strategies to fight COVID-19 worldwide: Challenges, recommendations, and a call for papers. J Med Internet Res. 2020; 22(6): e19284. PMID: 32501804 DOI: 10.2196/19284 [PubMed]

11. Ting DSW, Carin L, Dzau V, Wong TY. Digital technology and COVID-19. Nat Med. 2020; 26(4): 45961. PMID: 32284618 DOI: $10.1038 / \mathrm{s} 41591-020-$ 0824-5 [PubMed]

12. Ye J. The role of health technology and informatics in a global public health emergency: Practices and implications from the COVID-19 pandemic. JMIR Med Inform. 2020; 8(7): e19866. PMID: 32568725 DOI: 10.2196/19866 [PubMed]

13. Ye Q, Zhou J, Wu H. Using information technology to manage the COVID-19 pandemic: Development of a technical framework based on practical experience in China. JMIR Med Inform. 2020; 8(6): e19515. PMID: 32479411 DOI: 10.2196/19515 [PubMed]

14. Higgins JP. Cochrane handbook for systematic reviews of interventions version 5.0. 1 [Internet]. 
2008 [cited: 1 Oct 2020]. The Cochrane Collaboration. Available from: http://www cochrane-handbook org

15. Moher D, Shamseer L, Clarke M, Ghersi D, Liberati A, Petticrew M, et al. Preferred reporting items for systematic review and meta-analysis protocols (PRISMA-P) 2015 statement. Syst Rev. 2015; 4(1): 1. PMID: 25554246 DOI: $10.1186 / 2046-4053-4-1$ [PubMed]

16. Sahama T. Health informatics domain knowledge analysis: An information technology perspective. In: Croll P, Sintchenko V (Eds.). Frontiers of Health Informatics-Redefining Healthcare: Annual HISA Health Informatics Conference. Health Informatics Society of Australia; 2009.

17. Schuemie M, Talmon J, Moorman P, Kors J. Mapping the domain of medical informatics. Methods Inf Med. 2009; 48(1): 76-83. PMID: 19151887 DOI: 10.3414/me0576 [ [PubMed]

18. Ganjali R, Khoshrounejad F, Habibi MRM, Taherzadeh Z, Golmakani R, Mostafavi SM, et al. Effect and features of information technology-based interventions on self-management in adolescent and young adult kidney transplant recipients: A systematic review. Adolesc Health Med Ther. 2019; 10: 173-90. PMID: 31686939 DOI: 10.2147/AHMT.S200801 [PubMed]

19. Moher D, Liberati A, Tetzlaff J, Altman DG. Preferred reporting items for systematic reviews and metaanalyses: The PRISMA statement. BMJ. 2009; 339: b2535. PMID: 19622551 DOI: 10.1136/bmj.b2535 [PubMed]

20. Vandenbroucke JP, Von Elm E, Altman DG, Gøtzsche PC, Mulrow CD, Pocock SJ, et al. Strengthening the reporting of observational studies in epidemiology (STROBE): Explanation and elaboration. PLoS Med.
2007; 4(10): e297. PMID: 17941715 DOI: 10.1371/journal.pmed.0040297 [PubMed]

21. Moons KG, Wolff RF, Riley RD, Whiting PF, Westwood M, Collins GS, et al. PROBAST: A tool to assess risk of bias and applicability of prediction model studies: explanation and elaboration. Ann Intern Med. 2019; 170(1): W1-33. PMID: 30596876 DOI: 10.7326/M181377 [PubMed]

22. Estabrooks C, Goel V, Thiel E, Pinfold P, Sawka C, Williams I. Decision aids: Are they worth it? A systematic review. J Health Serv Res Policy. 2001; 6(3): 170-82. PMID: 11467275 DOI: $10.1258 / 1355819011927431$ [PubMed]

23. Estabrooks CA, Cummings GG, Olivo SA, Squires JE, Giblin C, Simpson N. Effects of shift length on quality of patient care and health provider outcomes: Systematic review. Qual Saf Health Care. 2009; 18(3): 181-8. PMID: 19467999 DOI: 10.1136/qshc.2007.024232 [ubMed]

24. Thomas J, Harden A. Methods for the thematic synthesis of qualitative research in systematic reviews. BMC Med Res Methodol. 2008; 8: 45. PMID: 18616818 DOI: 10.1186/1471-2288-8-45 [PubMed]

25. Niazkhani Z, Pirnejad H, Khazaee PR. The impact of health information technology on organ transplant care: A systematic review. Int J Med Inform. 2017; 100: 95-107. PMID: 28241943 DOI: 10.1016/j.ijmedinf.2017.01.015 [PubMed]

26. Shahmoradi L, Abtahi H, Amini S, Gholamzadeh M. Systematic review of using medical informatics in lung transplantation studies. Int J Med Inform. 2020; 136: 104096. PMID: 32058262 DOI: 10.1016/j.ijmedinf.2020.104096 [PubMed] 